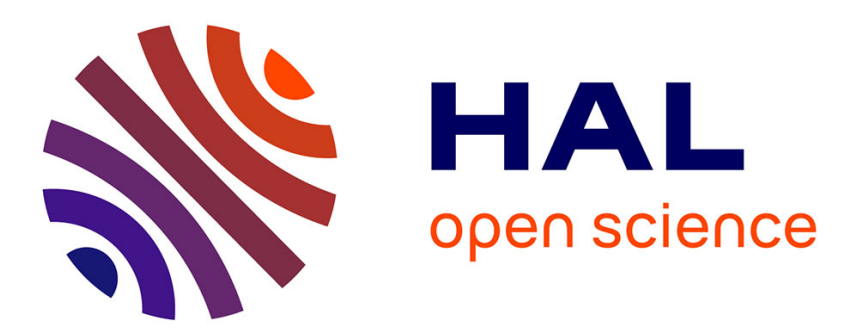

\title{
Quand la compétition électorale détermine la disproportionnalité : bonus majoritaire et élections régionales en France et en Italie \\ Camille Bedock
}

\section{- To cite this version: \\ Camille Bedock. Quand la compétition électorale détermine la disproportionnalité : bonus majori- taire et élections régionales en France et en Italie. Revue internationale de politique comparée, 2017, 24 (3), pp.233-255. 10.3917/ripc.243.0233 . hal-02266759}

\section{HAL Id: hal-02266759 \\ https://hal.science/hal-02266759}

Submitted on 16 Aug 2019

HAL is a multi-disciplinary open access archive for the deposit and dissemination of scientific research documents, whether they are published or not. The documents may come from teaching and research institutions in France or abroad, or from public or private research centers.
L'archive ouverte pluridisciplinaire HAL, est destinée au dépôt et à la diffusion de documents scientifiques de niveau recherche, publiés ou non, émanant des établissements d'enseignement et de recherche français ou étrangers, des laboratoires publics ou privés. 


\title{
Quand la compétition électorale détermine la disproportionnalité : bonus majoritaire et élections régionales en France et en Italie
}

Bedock Camille, «Quand la compétition électorale détermine la disproportionnalité : bonus majoritaire et élections régionales en France et en Italie ", Revue internationale de politique comparée, 2017/3 (Vol. 24), p. 233-255

\section{Résumé}

Cet article s'interroge sur les effets du changement des modalités de la compétition électorale sur le niveau de disproportionnalité enregistré dans un type bien particulier de système électoral mixte : le système proportionnel avec bonus majoritaire. Il s'intéresse aux cas français et italien à partir des trois dernières élections régionales qui ont eu lieu dans ces deux pays. Les deux systèmes partagent des caractéristiques importantes : ils utilisent un système proportionnel avec bonus et présentent tous les deux des systèmes partisans dans lesquels un troisième pôle électoral fort refusant toute alliance électorale a récemment émergé ou s'est considérablement renforcé, dans un contexte auparavant caractérisé par un bipolarisme fragmenté. À partir de la simulation de plusieurs scénarios de répartition des voix, de l'étude de statistiques descriptives et de régressions linéaires, l'article montre que la fragmentation en plusieurs pôles et le caractère plus ou moins serré de la compétition électorale contribuent de manière très significative au niveau de disproportionnalité enregistré. Plus largement, ce niveau croissant de disproportionnalité pose des questions importantes sur la qualité de la représentation en France et en Italie.

\begin{abstract}
This article investigates the effect of the changing format of electoral competition on the level of disproportionality in a peculiar type of mixed electoral system: PR with Bonus. It focuses on the way changing patterns of electoral competition affect the level of disproportionality across elections in countries using PR with bonus. The paper uses the cases of the three last regional elections in France and Italy to investigate this research question, as both countries share defining characteristics: they use PR with bonus in the regional elections, and they have both been characterized by the emergence or the surge of a strong third pole refusing any alliance with other parties in a party system that was previously characterized by fragmented bipolarism. Through electoral simulations, descriptive statistics and by way of an OLS regression we show that the fragmentation in several electoral poles and the closeness of the race in a given regional contest both contribute to a significant rise in the level of disproportionality, with consequences that are not to be discarded for the quality of representation in France and Italy.
\end{abstract}




\section{Introduction}

L'une des caractéristiques les plus cruciales à examiner pour comprendre l'effet des systèmes électoraux sur la compétition politique est la distorsion entre votes et sièges qu'ils impliquent. Comme le montre par exemple Michael Gallagher, l'écart moyen entre votes et sièges obtenus dans des pays utilisant des systèmes extrêmement proportionnels comme les Pays-Bas entre 1946 et 2012 a varié de 0,9 à 1,6 pour les élections législatives tandis que des pays utilisant des systèmes purement majoritaires comme le Royaume-Uni enregistraient une distorsion moyenne comprise entre 4,13 et 17,45 entre 1945 et 2015. En effet, si l'on peut considérer qu'un système électoral « idéal » serait celui qui concilierait une représentation très fidèle des préférences des électeurs avec la capacité à former un gouvernement responsable, la réalité implique nécessairement un arbitrage entre les deux objectifs (Carey et Hix 2011).

Les auteurs qui ont cherché à expliquer les déterminants de la disproportionnalité se sont focalisés de manière quasiment exclusive sur les effets des différents systèmes électoraux et de leurs caractéristiques, comme la magnitude, pour expliquer la traduction des votes en sièges (Cox 1997; Lijphart 1994; Rae 1969; Taagepera et Shugart 1989). L'influence des systèmes partisans sur les systèmes électoraux (Rokkan 1970; Boix 1999; Colomer 2005; Benoit 2002, 2007) et inversement (Duverger 1951; Cox 1997; Taagepera 2007) est désormais fermement établie. Les analyses récentes portant spécifiquement sur le niveau de disproportionnalité ont progressivement intégré les systèmes électoraux «complexes » (Bedock et Sauger 2014; Farrell et Katz 2014; Ferrara, Herron, et Nishikawa 2005; Moser et Scheiner 2004). Pour autant, les contributions existantes ne s'intéressent pas à la capacité de la structure de la compétition électorale à affecter de manière potentiellement très importante le degré de distorsion votes/sièges. Comment les caractéristiques de la compétition électorale peuvent-elles, dans certaines conditions, avoir un effet drastique sur le niveau de disproportionnalité enregistré ?

Cet article s'intéresse à cette question en abordant le cas des élections régionales en France et en Italie. Ces deux cas présentent ici un intérêt tout particulier. Tout d'abord, ils utilisent pour les élections régionales un système électoral atypique qui implique l'attribution d'un large bonus majoritaire à la coalition arrivée en tête, même si les modalités d'attribution du bonus diffèrent sensiblement. Ensuite, les deux pays sont caractérisés par une compétition électorale fragmentée, structurée précédemment autour de deux grands pôles ou deux grands partis en perte de vitesse du fait de l'émergence ou de l'affirmation récente d'un troisième pôle puissant: le Front National en France, et le mouvement Cinq étoiles en Italie. Un article portant sur ce même 
mode de scrutin avait déjà montré que les incitations stratégiques d'un mode de scrutin proportionnel avec bonus majoritaire tendent à favoriser un niveau de disproportionnalité intermédiaire entre ceux enregistrés dans les systèmes proportionnels et majoritaires. Le fait d'autoriser les coalitions pré-électorales amène à favoriser à la fois une compétition bipolaire et fragmentée (Bedock et Sauger 2014). Je m'attacherai ici à démontrer que la fragmentation entre différents pôles de la compétition électorale ainsi que la lutte entre les deux principales coalitions pour obtenir le bonus ont un effet important sur le niveau de disproportionnalité enregistré. En effet, le lien entre système électoral et disproportionnalité n'est pas nécessairement ni stable, ni linéaire : de par leurs effets mécaniques très sensibles au format de la compétition électorale, les systèmes électoraux avec bonus peuvent entraîner des niveaux de disproportionnalité très importants. Plus largement, les niveaux parfois vertigineux de distorsion votes/sièges enregistrés dans le cas italien et surtout dans le cas français posent la question de la capacité à représenter de manière équitable les votes des citoyens. Les cas italien et français mettent fortement en question la capacité de systèmes électoraux supposément conçus pour « assurer la majorité mais préserver les minorités (majority-assuring but minority-friendly, D'Alimonte 2015) à assurer une représentation politique juste dans les situations où il existe un pôle électoral puissant considéré comme un partenaire de coalition inacceptable par les autres partis. Alors que les systèmes mixtes sont censés représenter «le meilleur des deux mondes » (Shugart et Wattenberg 2001) représentativité et gouvernabilité - on montre au contraire qu'ils peuvent parfois encourager des niveaux de disproportionnalité plus drastiques même que les systèmes majoritaires.

Dans un premier temps, on reviendra brièvement sur les conséquences anticipées dans la littérature des systèmes mixtes sur la disproportionnalité en général. Les cas italien et français seront ensuite présentés dans un second temps, en rappelant d'une part les principales caractéristiques des systèmes électoraux utilisés aux élections régionales, puis les récentes évolutions du système partisan des deux pays. Dans un troisième temps, deux hypothèses portant respectivement sur l'effet du troisième pôle puis sur le degré de compétitivité de la «course » entre les deux coalitions principales sur la disproportionnalité seront présentées. La quatrième section présentera une série de simulations électorales sur les effets mécaniques de ces deux systèmes en fonction de la fragmentation des différents pôles et du niveau de compétition. La dernière section propose une démonstration empirique basée sur la présentation des principales statistiques descriptives sur l'évolution des caractéristiques de la compétition électorale aux régionales en France et en Italie et sur une régression linéaire. L'ensemble de ces analyses confirment l'effet indépendant et conjoint de la fragmentation en plus de deux pôles et de la compétitivité sur le niveau de disproportionnalité enregistré. 


\section{Section 1- Systèmes mixtes et disproportionnalité}

Louis Massicotte et André Blais ont défini les systèmes électoraux mixtes comme des systèmes dont les «mécanismes impliquent la combinaison de différentes formules électorales (majorité relative ou absolue, proportionnelle) pour une même élection » (1999, 345). Cette définition est dans une certaine mesure insatisfaisante pour décrire les systèmes proportionnels avec bonus comme ceux utilisés en France et en Italie pour les élections régionales, puisqu'ils n’impliquent pas nécessairement l'usage de deux formules électorales différentes. Cependant, l'intégration d'un bonus majoritaire dans un système proportionnel correspond bien à l'idée de combiner des principes proportionnels et majoritaires dans le même système électoral. C'est pourquoi on considèrera dans la suite de cet article que ces systèmes sont bien des systèmes électoraux mixtes, bien qu'il s'agisse de systèmes mixtes «non conventionnels » (Bochsler et Bernauer 2014). Les contributions portant spécifiquement sur l'effet des systèmes électoraux mixtes sur le niveau de disproportionnalité sont rares, et ont tendu à se focaliser sur les systèmes où les électeurs sont amenés à exprimer deux votes. Leurs conclusions ne sont donc pas directement applicables aux systèmes proportionnels avec bonus majoritaires, bien qu'elles donnent des éléments de compréhension précieux pour expliquer les niveaux de disproportionnalité enregistrés dans ces systèmes. Elles se penchent sur deux grands types de facteurs: les effets mécaniques complexes des systèmes électoraux mixtes d'une part, et les incitations stratégiques ambiguës qu'ils entraînent d'autre part.

S'intéressant à l'effet de multiples variations des systèmes électoraux mixtes sur le nombre de candidats et de partis en compétition au niveau des circonscriptions électorales, Robert G. Moser et Ethan Sheiner relativisent l'hypothèse dite de «contamination » (Ferrara, Herron, et Nishikawa 2005; Herron et Nishikawa 2001). Ils montrent que la part proportionnelle et majoritaire des scrutins mixtes ont des effets indépendants et distincts sur la fragmentation et sur la disproportionnalité. Les systèmes mixtes qui utilisent des mécanismes de compensation entre le niveau majoritaire et proportionnel enregistrent des niveaux de disproportionnalité plus faibles, tandis que les systèmes requérant la majorité absolue des votes dans la part majoritaire sont plus disproportionnels que ceux qui requièrent une majorité relative (Moser et Scheiner 2004). En se concentrant spécifiquement sur le cas des systèmes proportionnels avec bonus majoritaire, Camille Bedock et Nicolas Sauger montrent que le facteur central à analyser pour comprendre le niveau de distorsion entre votes et sièges est celui du mode d'allocation du bonus majoritaire. Ainsi, l'existence d'un bonus fixe plutôt que variable renforce la disproportionnalité. Les deux 
auteurs montrent aussi que le niveau de bipolarisation limite le niveau de disproportionnalité enregistré (Bedock et Sauger 2014).

Une autre série de recherches portant sur l'effet des systèmes électoraux mixtes sur les principales caractéristiques de la compétition s'est penchée sur les incitations stratégiques complexes qu'ils impliquent. R.G. Moser et E. Scheiner insistent par exemple sur l'importance des stratégies partisanes en montrant que le niveau variable d'institutionnalisation des partis politiques tempère les effets mécaniques des systèmes mixtes et peut entraîner des conséquences inattendues comme la prolifération de candidats dans la part majoritaire de l'élection (Moser et Scheiner 2004). Daniel Bocschler a quant à lui étudié une série de pays utilisant des systèmes mixtes compensatoires (l'Italie, l'Albanie, le Lesotho et le Venezuela), dans lesquels les partis obtenant de nombreux sièges dans la part majoritaire sont normalement sanctionnés dans la part proportionnelle du scrutin. Il montre les tactiques utilisées par les partis pour limiter cette compensation, notamment en incitant les électeurs à formuler deux votes distincts pour élargir leur majorité, ce qui entraîne une hausse de la disproportionnalité enregistrée (Bochsler 2012). A partir cette fois du cas hongrois utilisant un système mixte avec transferts de vote positifs, D. Bochsler montre que le niveau de disproportionnalité dépend de l'interaction entre le nombre de sièges compensatoires et le niveau de fragmentation (Bochsler 2014). Philip Manow fait des conclusions similaires à partir du cas allemand en montrant comment la part proportionnelle du scrutin peut exacerber le niveau de disproportionnalité enregistré dans le cas où beaucoup de petits partis se présentent dans la part majoritaire dans des circonscriptions dans lesquelles ils n'ont aucune chance de l'emporter (Manow 2011). De la même manière, le cas italien a montré comment les principaux partis ont été capables de détourner les mécanismes de compensation du scorporo dans le système électoral utilisé entre 1993 et 2005 (D'Alimonte et Bartolini 2002; D'Alimonte 2005). Dans une étude comparative et quantitative, Pedro Riera montre que le niveau de coordination électorale varie selon le type de système électoral utilisé et selon l'âge de la démocratie (2013). Enfin, plusieurs études portant sur des comparaisons franco-italiennes étudiant les systèmes proportionnels avec bonus ont montré à quel point le bonus majoritaire constitue une incitation majeure pour les partis politiques à se coordonner dans des coalitions inclusives, et ce d'autant plus dans un contexte fragmenté (Bedock et Sauger 2014; Di Virgilio, Dolez, et Laurent 2016). En résumé, comme le notent Aldo Di Virgilio, Bernard Dolez et Annie Laurent, «quand la règle électorale est 'complexe', la contrainte qu'elle exerce sur les acteurs est elle aussi complexe ; en retour, les acteurs peuvent plus aisément en jouer» $(2016,367)$.

Ces quelques contributions mettent en exergue plusieurs points importants. Premièrement, le niveau de disproportionnalité enregistré dans les systèmes électoraux mixtes est 
supposé se situer à un niveau intermédiaire entre celui des pays utilisant la proportionnelle et ceux utilisant les systèmes majoritaires. Ce niveau intermédiaire dépend lui-même des types de mécanismes compensatoires ou des modes d'allocation du bonus majoritaire quand celui-ci existe. Deuxièmement, les systèmes mixtes impliquent de par leur complexité des incitations contradictoires et une marge de manœuvre importante pour les partis en compétition, qui peuvent «tempérer» les effets mécaniques du système électoral. Toutes ces contributions suggèrent en filigrane que les systèmes électoraux mixtes ont non seulement des effets plus imprévisibles que les systèmes électoraux proportionnels et majoritaires sur la disproportionnalité, mais aussi plus changeants. C'est donc à ce titre que l'on peut s'interroger sur l'effet propre du changement du format de la compétition électorale sur la distorsion votes / sièges.

\section{Section 2 - La tripolarisation des systèmes partisans français et italien}

Avant de présenter les hypothèses et l'analyse empirique, il est important de rappeler brièvement quelles sont les principales caractéristiques des systèmes partisans français et italien ainsi que les systèmes électoraux utilisés pour les élections régionales. Les deux pays s'avèrent particulièrement intéressants pour comprendre les conséquences du format changeant de la compétition électorale sur la disproportionnalité parce qu'ils partagent des caractéristiques clés : des systèmes partisans tripolaires et fragmentés avec un pôle électoral refusant les alliances avec les autres partis et des systèmes électoraux proportionnels avec bonus majoritaire.

L'Italie est un des seuls pays du monde dans lequel la plupart des systèmes électoraux utilisés au niveau local et national constituent des variantes de systèmes proportionnels avec bonus majoritaire. La question du système électoral national se pose à nouveau après la décision de la Cour constitutionnelle italienne de janvier 2016 d'annuler plusieurs dispositions de l'Italicum adopté en 2015 pour remplacer le système proportionnel avec bonus utilisé lors des élections de 2006, 2008 et 2013 à la Chambre des députés et au Sénat. Il est possible, à partir de l'exemple de ces trois élections nationales organisées avec la loi électorale dite du Porcellum prévoyant un bonus majoritaire substantiel au niveau national à la Chambre et régional au Sénat de proposer quelques pistes sur les conséquences de l'émergence d'un troisième pôle sur la disproportionnalité. Après avoir été caractérisé depuis 1993 par un bipolarisme dit «fragmenté » (Chiaramonte 2010) voire « déstructuré » (Pasquino et Valbruzzi 2013), une forte volatilité ${ }^{1}$ et un renouvellement quasipermanent des alternatives partisanes à gauche comme à droite entre 1993 et 2008, le système

${ }^{1}$ Celle-ci atteint des records en 1994 avec une volatilité de 36,7\% et en 2013 où elle s'élève à 41,3\%. 
partisan italien s'est vu totalement transformé après les élections législatives de 2013. Cette évolution a ensuite été confirmée lors les élections régionales qui se sont déroulées entre 2013 et 2015 en Italie (Tronconi 2015). Alors que les élections nationales et locales étaient depuis une quinzaine d'années caractérisées par l'affrontement de deux pôles - l'un de centre-droit, l'autre de centre-gauche - qui alternaient au pouvoir, le Movimento Cinque Stelle a émergé en 2013 comme un acteur politique incontournable, obtenant près de 25,6\% des votes à la Chambre basse, faisant chuter le total des votes obtenus par les deux principaux partis à seulement 58,7\% en 2013 (Chiaramonte et Emanuele 2013).

L'émergence de ce troisième pôle n'a pas été sans conséquences sur la disproportionnalité enregistrée aux élections depuis 2013. Premièrement, la coalition de centre-gauche, arrivée en tête aux élections à la Chambre des députés a obtenu grâce au bonus majoritaire près de $54 \%$ des sièges avec seulement 29,6\% des votes, à peine $0,4 \%$ de plus que la coalition de centre-droit. Le niveau de disproportionnalité enregistré avec l'indice de Gallagher s'élevait donc à 17,3 soit trois fois plus qu'aux élections précédentes. Ce résultat suggère fortement le potentiel très distorsif des primes majoritaires quand le parti arrivé en tête obtient un score bien en deçà de 50\% des votes, dans un contexte de très forte fragmentation (Conti et Memoli 2015; Passarelli 2014). De tels niveaux de disproportionnalité ne peuvent être comparés qu'avec ceux enregistrés dans des systèmes majoritaires comme la France ou le Royaume-Uni. D'autre auteurs soulignent que le niveau de disproportionnalité est lié à l'écart infinitésimal entre les deux principales coalitions (Baldini 2013). Finalement, il est très clair que c'est le troisième pôle, ici le M5S, qui a été le plus sévèrement pénalisé par le système électoral en termes absolus, surtout si on le compare avec des petites formations politiques qui avaient choisi d'intégrer les coalitions de centre-droit et de centre-gauche (Conti et Memoli 2015, 530). Il est aussi important de rappeler que le système partisan italien est caractérisé par un processus de désinstitutionnalisation et de dé-nationalisation (Vampa 2015, 2016), une tendance que les très récentes scissions du Parti Démocrate en mars 2017 ne font que confirmer. Ces deux dynamiques sont particulièrement prononcées dans les élections régionales qui se caractérisent par des systèmes partisans régionaux de plus en plus différenciés (Tronconi et Roux 2009).

Le système électoral utilisé en Italie dans la plupart des régions ordinaires - bien qu'un grand nombre d'entre elles aient désormais adopté des variations plus ou moins substantielles qu'on ne présentera pas ici mais qui seront incorporées dans nos modèles empiriques ${ }^{2}$ - a été mis

\footnotetext{
${ }^{2}$ Les variations à la loi Tatarella adoptées dans les régions ordinaires de Toscane, dans les Pouilles, en Calabre, en Campanie, en Vénétie, en Emilie-Romagne, en Ombrie, dans les Abruzzes et dans les Marches
} 
en place en 1995 avec la loi dite Tatarella qui instituait également l'élection directe des présidents de région. Les électeurs ont deux votes : un vote de liste au niveau provincial et un vote pour choisir un candidat à la présidence de la région élu avec un système majoritaire. Les candidats à la présidence sont à la tête d'une liste régionale et l'ensemble des listes provinciales doivent être reliées à un candidat à la présidence. $80 \%$ des sièges sont alloués aux listes provinciales à la proportionnelle, avec un seuil de 3\% ou l'obligation d'être lié à une coalition obtenant au moins $5 \%$ des votes. $20 \%$ des sièges sont alloués aux listes régionales. Si le vainqueur à la présidence de la région est élu avec moins de 50\% des votes, l'ensemble des sièges alloués à la liste régionale lui reviennent. Si le vainqueur à la présidence obtient plus de 50\% des votes, la liste qu'il dirige obtient $10 \%$ des sièges de l'assemblée tandis que les $10 \%$ restants sont distribués proportionnellement. Les listes soutenant le candidat vainqueur à la présidence ont la garantie d'obtenir 55\% des sièges si les listes provinciales de la coalition obtiennent moins de $40 \%$ des votes, $60 \%$ dans le cas contraire. Enfin, si le nombre de sièges à distribuer est inférieur aux seuils de 55 ou $60 \%$, de nouveaux sièges sont créés jusqu'à ce que ces seuils soient atteints (Bedock et Sauger 2014, 102). Pour résumer, le système standard utilisé dans les régions ordinaires utilise plusieurs seuils de répartition, le bonus vise à assurer une majorité tout en étant compensatoire et deux formules électorales distinctes sont utilisées (l’une majoritaire, l'autre proportionnelle).

Comme c'est le cas en Italie, la France a longtemps été caractérisée par un « multipartisme bipolaire » (Bornschier et Lachat 2009) avec deux pôles principaux alternant au pouvoir au niveau local et au niveau national dans un contexte de forte fragmentation (Haegel 2007). Contrairement à la situation italienne entre 1993 et 2008 cependant, les deux principaux partis français (le Parti Socialiste et les Républicains, anciennement l'UMP) ont dû faire face dès les années 1980 à la concurrence électorale de l'extrême droite du Front National. Cependant, le système majoritaire à deux tours en place au niveau national pour les élections présidentielles et législatives a toujours fortement limité l'accès du Front National à tout mandat électif. De la même façon, le fait que les systèmes électoraux au niveau local favorisent systématiquement les partis capables de gagner au second tour et donc de construire des alliances électorales ou d'attirer des électeurs d'autres partis a constitué une difficulté majeure pour le Front National en vue d'obtenir des élus locaux (Sauger 2007). De plus, les différents systèmes électoraux français impliquent une coordination préélectorale complexe et la constitution de coalitions dont le Front National est systématiquement exclu (Di Virgilio, Dolez, et Laurent 2016). L'électorat français a longtemps été qualifié de « tripartite » (Grunberg et Schweisguth 2003), avec des différences idéologiques importantes entre

partagent toutes les caractéristiques essentielles de la loi Tatarella, à savoir plusieurs seuils de distribution des sièges, un bonus compensatoire et l'existence de deux formules électorales distinctes. 
les électeurs du Parti Socialiste, des Républicains et du Front National limitant très fortement les flux entre les différents blocs. Cet état de fait a fortement changé dans la dernière décennie. Depuis 2007, les frontières des différents électorats, et plus spécifiquement ceux de l'électorat $\mathrm{LR} / \mathrm{FN}$ ont tendu à disparaitre alors que les partis de droite ont de plus en plus concentré leurs discours sur des questions comme l'immigration (Gougou et Labouret 2013). De plus, le Front National s'est révélé de plus en plus souvent capable de constituer non pas le troisième parti, mais aussi le second voire le premier dans certaines consultations électorales. La part des votes obtenus par le Front National a été croissante : 17,9\% des votes au premier tour de l'élection présidentielle en 2012, 24,8\% aux élections européennes de 2014 (ce qui en faisait alors le plus gros parti), en tête dans 71 départements aux élections départementales de 2015 et 28,4 en moyenne au premier tour des élections régionales de 2015 (Ehrhard 2016). L'élection présidentielle de 2017 semble suggérer que le système partisan pourrait même être en train de devenir plus éclaté encore, avec quatre pôles électoraux autour des 20\%, un Parti Socialiste en déshérence et des scénarios de consolidation encore très incertains (Gougou et Persico 2017).

Depuis 2003, la France utilise un système électoral à deux tours proportionnel avec bonus majoritaire pour élire les conseillers régionaux pour une durée de six ans. En 2013, après l'abrogation d'une loi qui visait à supprimer les conseillers régionaux et départementaux pour les remplacer par des conseillers territoriaux, une nouvelle loi a significativement réduit le nombre de régions française qui passe de 22 à 13 régions ${ }^{3}$. Les listes sont en compétition au niveau régional. Si une liste obtient la majorité absolue des voix au premier tour, elle obtient $25 \%$ des sièges alors que les $75 \%$ des sièges restants sont distribués à la proportionnelle, y compris à la liste gagnante, à l'ensemble des partis ayant obtenu au moins 5\% des votes. Si aucune liste n'obtient la majorité absolue, un second tour est organisé dans lequel seules les listes ayant obtenu plus de 10\% des suffrages exprimés au premier tour sont autorisées à concourir. Les listes ayant obtenu entre 5 et $10 \%$ des suffrages au premier tour ont la possibilité de fusionner avec les listes présentes au second tour. La liste qui obtient la majorité des voix reçoit $25 \%$ des sièges alors que le reste des sièges sont répartis proportionnellement entre l'ensemble des listes ayant obtenu au moins 5\% des votes, liste gagnante comprise. Le système électoral régional français est donc bien distinct du système italien : il n'y a qu'un seuil de répartition des sièges, le bonus est fixe ( $25 \%$ des sièges) et

\footnotetext{
${ }^{3}$ Seules les frontières des régions Île de France, Bretagne, Centre-Val de Loire, Corse, Pays de la Loire and Provence-Alpes-Côte d'Azur sont restées inchangées. Sept nouvelles régions ont été créées par la fusion de deux ou trois anciennes régions selon les cas : Nouvelle Aquitaine, Occitanie, Auvergne-Rhône Alpes, Normandie, Hauts-de-France, Bourgogne-Franche-Comté et Grand-est. Pour des raisons de comparabilité, la Corse a été exclue de l’analyse.
} 
les seuils électoraux pour accéder à la répartition des sièges sont considérablement plus élevés qu'en Italie.

\section{Section 3 - Hypothèses}

Des recherches précédentes ont déjà établi que des seuils de représentation plus élevés combinés avec un bonus fixe expliquent pourquoi la disproportionnalité est bien supérieure dans le cas français (Bedock et Sauger 2014). On veut ici discuter des effets mécaniques sur la disproportionnalité de l'émergence d'un troisième pôle refusant la coordination pré-électorale sur les niveaux de disproportionnalité enregistrés an nivean régional. Ainsi, les deux hypothèses suivantes cherchent à expliquer les différences entre régions plutôt qu'entre pays.

\section{H1. Plus le troisième pôle obtient de votes, plus le niveau de disproportionnalité enregistré est élevé.}

Comme nous l'avons déjà mentionné, en se basant sur les résultats de 2013 aux élections législatives en Italie, on peut s'attendre à ce que le niveau de disproportionnalité soit plus élevé lorsqu'il y a un troisième pôle électoral puissant que quand seuls deux partis ou coalitions sont en compétition pour l'obtention du bonus majoritaire. Le raisonnement sous-jacent à cette hypothèse est simple : il n'y a qu'un « prix » (le bonus majoritaire), et ce prix ne peut aller qu'à un seul parti ou à une seule coalition. Autrement dit, et quelle que soit la répartition des sièges entre les différentes forces en présence, il ne peut y avoir qu'un gagnant. Au contraire, il peut y avoir un seul ou de multiples perdants, c'est-à-dire l'ensemble des partis ou des coalitions qui n'obtiennent pas le bonus, et qui sont d'autant plus pénalisées quand la " punition » associée à la non-obtention du prix est distribuée entre plusieurs concurrents.

H2. Plus la compétition en vue de l'obtention du bonus est serrée entre les deux principaux partis ou coalitions, plus le niveau de disproportionnalité enregistré est élevé.

À nouveau, cette hypothèse est notamment basée sur l'extrapolation des résultats des élections législatives de 2013 en Italie pendant laquelle la « course » entre le centre-gauche et le centre-droit en vue de l'obtention du bonus à la Chambre des députés avait été particulièrement disputée. On s'attend à ce que, quand la compétition entre les deux principales forces électorales dans une région donnée est serrée, le niveau de distorsion votes/sièges soit plus élevé. À nouveau, cette attente est liée au fait qu'il ne peut y avoir qu'un seul gagnant dans les systèmes électoraux avec bonus. Lorsque l'élection est très disputée, cela signifie concrètement que la coalition ou le parti 
gagnant obtient l'intégralité du prix avec seulement quelques votes de plus que son principal adversaire.

A ces deux hypothèses, il faut ajouter un questionnement important. Il s'agit non seulement de vérifier ces attentes, mais aussi à quel point ces deux situations (séparément voire en combinaison) peuvent avoir un potentiel de distorsion important sur le niveau de disproportionnalité. Ainsi, il s'agit de savoir si les variations du niveau de disproportionnalité sont existantes mais négligeables, ou si elles sont au contraire potentiellement suffisamment élevées pour que la contrainte constituée par ces systèmes électoraux pour accéder aux sièges puisse être considérée comme problématique pour la bonne représentation des opinions des électeurs.

\section{Section 4- Simulations sur les effets de la fragmentation des pôles électoraux et du niveau de compétitivité sur la disproportionnalité}

Pour évaluer les effets mécaniques indépendants de la fragmentation des pôles électoraux et du niveau de compétitivité sur le niveau de disproportionnalité, nous avons testé une série de scénarios dans des simulations électorales. Ces scénarios font varier d'une part le nombre de blocs en compétition, et d'autre part l'écart entre les deux blocs arrivés en tête, afin de mesure le niveau de disproportionnalité qui en résulte, mesuré classiquement par l'indice des moindres carrés de Gallagher (1991). A partir d'une circonscription fictive avec 2250000 votes exprimés dans laquelle 60 sièges doivent être distribués, nous avons calculé le niveau de disproportionnalité enregistrés respectivement en France et en Italie dans six scénarios: deux blocs avec une compétition équilibrée (1), deux blocs avec une compétition déséquilibrée (2), trois blocs avec une compétition équilibrée (3), trois blocs avec une compétition déséquilibrée (4), quatre blocs avec une compétition équilibrée (5) et quatre blocs avec une compétition déséquilibrée (6). Comme mentionné précédemment, le bonus est fixe dans le cas français (25\%) alors que les 75\% de sièges répartis à la proportionnelle le sont avec le système de la plus forte moyenne. Dans le cas italien, pour des raisons de simplicité, nous avons réparti l'ensemble des sièges avec la méthode Hagenbach-Bishoff qui est utilisée pour répartir les sièges au niveau provincial, et nous avons supposé que les votes obtenus dans le scrutin de liste sont identiques aux votes obtenus par le candidat président auxquels sont rattachées les listes dans la part majoritaire. Comme nous l'avons déjà évoqué, le bonus est variable : il est compris entre 10 et 20\% selon les votes obtenus par les listes, le bloc gagnant devant obtenir selon ses votes au moins $55 \%$ ou au moins $60 \%$ des sièges. Pour cette raison, le total des sièges distribués dans le cas italien selon les scénarios peut dépasser 60 (cf. Annexes). 
Table 1. Simulations du niveau de disproportionnalité enregistré pour 12 scénarios

\begin{tabular}{|l|c|c|}
\hline Scénarios testés & France & Italie \\
\hline $\begin{array}{l}\text { Deux blocs, compétition équilibrée } \\
\text { (Bloc A : 51\%, Bloc B : 49\%) }\end{array}$ & 12,3 & 5,6 \\
\hline $\begin{array}{l}\text { Deux blocs, compétition déséquilibrée } \\
\text { (Bloc A : 65\%, Bloc B : 35\%) }\end{array}$ & 8,3 & 3,3 \\
\hline $\begin{array}{l}\text { Trois blocs, compétition équilibrée } \\
\text { (Bloc A : 35\%, Bloc B : 33\%, Bloc C : 32\%) }\end{array}$ & 14,4 & 13,4 \\
\hline $\begin{array}{l}\text { Trois blocs, compétition déséquilibrée } \\
\text { (Bloc A: } 50 \%, \text { Bloc B : 26\%, Bloc C : 24\%) }\end{array}$ & 10,1 & \\
\hline $\begin{array}{l}\text { Quatre blocs, compétition équilibrée } \\
\text { (Bloc A : 27\%, Bloc B : 26\%, Bloc C : 24\%, } \\
\text { Bloc D: } 23 \% \text { ) }\end{array}$ & 14,7 \\
\hline $\begin{array}{l}\text { Quatre blocs, compétition déséquilibrée } \\
\text { (Bloc A: } 40 \%, \text { Bloc B: } 22 \%, \text { Bloc C: } 20 \%, \text { Bloc } \\
\text { D: } 18 \%)\end{array}$ & & 23 \\
\hline
\end{tabular}

Les résultats des simulations (Table 1) font apparaître trois résultats confirmant les deux hypothèses énoncées précédemment et permettant de les affiner. Premièrement, les niveaux de disproportionnalité enregistrés en France sont supérieurs à ceux enregistrés en Italie lorsque la compétition a lieu entre deux et trois blocs, mais pas lorsque quatre blocs sont en compétition. La différence de disproportionnalité est marquée lorsque deux blocs sont en compétition. Dans les scénarios où trois blocs s'affrontent, les différences entre les deux pays sont moins fortes. Lorsque quatre blocs sont en compétition, l'Italie enregistre des niveaux de disproportionnalité plus importants que ceux de la France. Ce résultat s'explique par le grand nombre de sièges qu'il faut « créer » pour assurer 55\% des sièges au bloc A. Autrement dit, la fragmentation en blocs a des effets encore plus marqués sur la disproportionnalité dans le cas italien, notamment lorsque la compétition entre les différents blocs est équilibrée.

Deuxièmement, les niveaux de disproportionnalité enregistrés sont notablement plus élevés lorsque trois et quatre blocs sont en compétition que dans les scénarios où deux blocs s'affrontent. Dans les deux pays, le niveau de disproportionnalité maximale est enregistré dans le scénario où quatre blocs s'affrontent dans une compétition équilibrée. Ces résultats suggèrent que la fragmentation en plus de trois blocs contribuerait encore à augmenter la disproportionnalité. De plus, les simulations confirment la première hypothèse : lorsque le troisième bloc est puissant, la disproportionnalité est plus élevée que dans le cadre d'une compétition bipolaire. 
Troisièmement, le niveau de disproportionnalité prévu dans nos simulations est systématiquement plus élevé lorsque la compétition est serrée que lorsqu'elle est déséquilibrée en faveur du bloc A. Ces résultats suggèrent que la seconde hypothèse est vérifiée. L'effet est bien plus marqué dans le cas italien, ce qui s'explique à nouveau par les règles de distribution des sièges imposant à la coalition gagnante d'avoir au moins 55\% des sièges de l'assemblée.

\section{Section 5- Une analyse empirique des effets de la fragmentation des pôles et du niveau de compétition sur la disproportionnalité}

\section{Analyse descriptive}

Avant de commenter les résultats des régressions linéaires conduites pour confirmer et étayer les résultats des simulations, il est utile de faire un détour par les statistiques descriptives sur une série d'indicateurs de la compétition électorale pour les trois dernières élections régionales ayant eu lieu en France et en Italie (Tables 2 et 3) ${ }^{4}$.

Dans le cas italien, plusieurs indicateurs de la compétition électorale sont restés stables (Table 2). L'écart moyen entre les deux principales coalitions dans chaque région est en moyenne élevé, compris entre 16,3 et 19,6\%. Les déviations à la moyenne sont très importantes, suggérant que le niveau moyen de compétitivité dans chaque région italienne est extrêmement variable. Cela illustre le phénomène de dé-nationalisation de la compétition politique évoqué plus haut. Le nombre moyen de listes en compétition obtenant au moins un pour cent des votes et le nombre effectif de partis obtenant des votes (NeffV) sont restés stables pendant les trois élections, avec en moyenne entre 10 et 12 listes obtenant au moins un pour cent des votes et un nombre effectif de partis compris entre 5,7 et 6,7. En moyenne, le parti arrivé en tête dans chaque région a obtenu environ 30\% des votes à chaque élection. L'ensemble de ces indicateurs suggère que la compétition électorale est particulièrement fragmentée, aucun parti n'étant en mesure à lui tout seul d'obtenir une majorité absolue des voix.

Ce qui a changé au contraire très fortement entre 2005 et 2015, c'est le niveau de bipolarisation et, bien évidemment et symétriquement, la force relative moyenne du troisième pôle. En 2005 et 2010, les coalitions de centre-gauche et centre-droit ont été en mesure, en moyenne, de s'arroger la quasi-totalité des voix dans la part majoritaire du scrutin : 97 et 95\% respectivement. Les indicateurs de dispersion montrent, de plus, que cette situation de

\footnotetext{
${ }^{4}$ Il est à noter que le «troisième pôle ", puisqu'il est défini en fonction de l'appartenance idéologique et non en fonction de son poids électoral, peut très bien être arrivé en tête dans certaines circonscriptions. De la même façon, l'écart entre les deux principaux partis ou coalitions peut concerner l'écart entre une des coalitions « traditionnelles » et le troisième pôle.
} 
bipolarisation quasi-parfaite était présente dans la totalité des régions italiennes ordinaires. Lors des scrutins régionaux qui se sont tenus entre 2013 et 2015, au contraire, le troisième pôle a réussi en moyenne à obtenir $27 \%$ des voix, et jusqu'à $40 \%$ dans les Marches. Ce nombre a donc été multiplié par huit en comparaison avec l'élection de 2015. Le niveau de disproportionnalité a également continuellement progressé. La distorsion votes/sièges moyenne était de 4,6\% en 2005, 6,6\% en 2010, et pas moins de 9,6\% pour les scrutins régionaux organisés entre 2013 et 2015 (avec un maximum de 13,9\% dans les Marches). Les indicateurs de dispersion suggèrent également que les différences entre régions en termes de disproportionnalité enregistrée ont tendu à devenir plus élevées à travers le temps. 
Table 2. Statistiques descriptives des principales caractéristiques de la compétition électorale aux élections régionales en Italie, 2005-2015

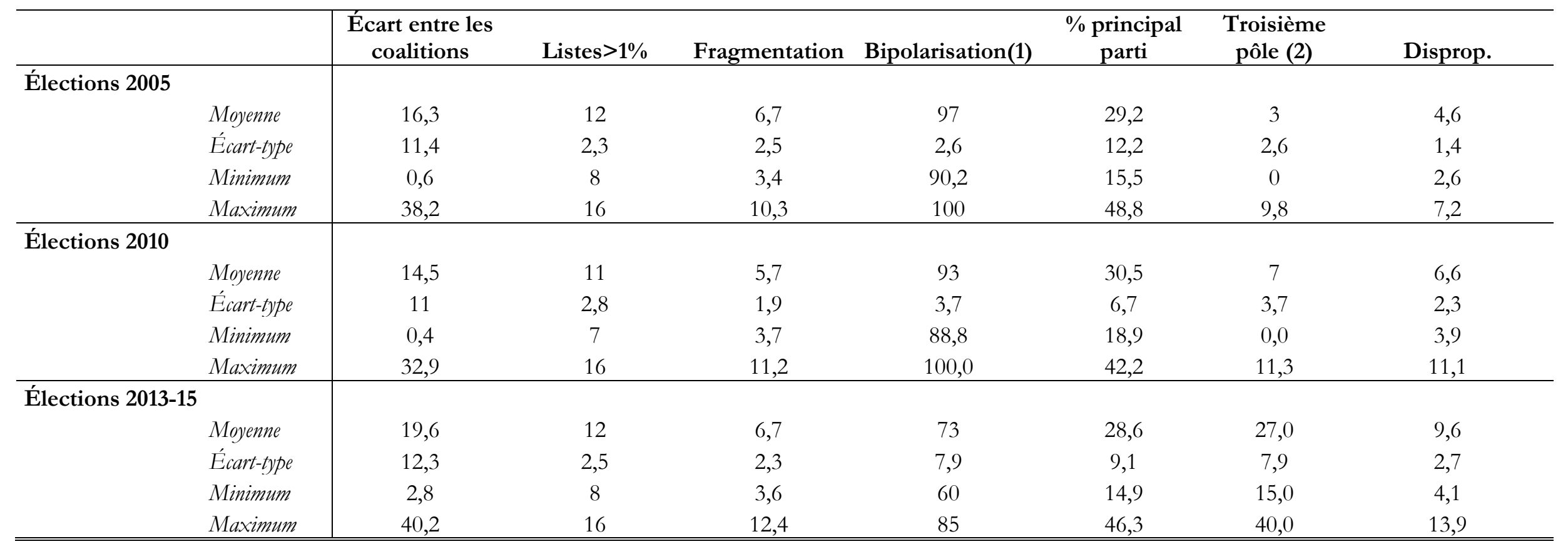

Note: Tous les calculs ont été effectués en prenant en compte les résultats dans les régions ordinaires suivantes : Piémont, Lombardie, Vénétie, Ligurie, Emilie-Romagne, Toscane, Ombrie, Marches, Lazio, Campanie, Abruzzes, Molise, Pouilles, Basilicata, Calabre.

(1) Ce chiffre a été obtenu en additionnant les scores obtenus par les coalitions de centre-gauche et de centre-droit dans la part majoritaire.

(2) Ce chiffre correspond à l'ensemble des votes obtenus par des partis n'appartenant ni à la coalition de centre-gauche ni de centre-droit dans la part majoritaire. 
Table 3. Statistiques descriptives des principales caractéristiques de la compétition électorale aux élections régionales en France, 2004-2015

\begin{tabular}{|c|c|c|c|c|c|c|c|c|c|}
\hline & & $\begin{array}{c}\text { Ecart entre les } \\
\text { coalitions }\end{array}$ & Listes $>1 \%$ & Fragmentation & $\begin{array}{c}\text { Bipolarisation } \\
\text { (1 } \text { (er }^{\text {er }} \text { (our) }\end{array}$ & $\begin{array}{c}\text { Bipolarisation } \\
\left(2^{\text {nd }} \text { tour }\right)\end{array}$ & $\begin{array}{l}\% \text { principal } \\
\text { parti (1) }\end{array}$ & $\begin{array}{l}\text { Troisième } \\
\text { pôle (2) }\end{array}$ & Disprop. \\
\hline \multicolumn{10}{|c|}{ (2: } \\
\hline & Moyenne & 13,7 & 7,6 & 4,4 & 59,9 & 87,5 & 35,0 & 12,8 & 11,5 \\
\hline & Écart-type & 6,8 & 1,4 & 0,6 & 6,8 & 7,1 & 4,6 & 7,2 & 0,8 \\
\hline & Minimum & 2,1 & 5,0 & 2,9 & 47,8 & 78,0 & 27,9 & 0,0 & 9,4 \\
\hline & Maximum & 24,0 & 10,0 & 5,7 & 79,2 & 100,0 & 46,3 & 22,0 & 13,3 \\
\hline \multicolumn{10}{|l|}{ Élections 2010} \\
\hline & Moyenne & 17,7 & 8,9 & 4,7 & 58,5 & 87,8 & 33,0 & 12,4 & 11,3 \\
\hline & Écart-type & 7,5 & 2,1 & 1,1 & 14,4 & 19,9 & 8,7 & 7,5 & 2,6 \\
\hline & Minimum & 5,8 & 7,0 & 3,8 & 48,1 & 77,1 & 26,4 & 0,0 & 8,1 \\
\hline & Maximum & 35,5 & 11,0 & 5,5 & 68,4 & 100,0 & 41,0 & 21,0 & 13,6 \\
\hline \multicolumn{10}{|l|}{ Élections 2015} \\
\hline & Moyenne & 8,2 & 8,1 & 4,3 & 60,0 & 85,0 & 33,9 & 30,0 & 22,9 \\
\hline & Écart-type & 6,7 & 0,7 & 0,3 & 4,2 & 10,3 & 4,0 & 9,7 & 6,6 \\
\hline & Minimum & 0,3 & 7,0 & 3,7 & 55,4 & 67,6 & 27,9 & 14,0 & 14,1 \\
\hline & Maximum & 21,7 & 9,0 & 4,8 & 68,5 & 100,0 & 40,6 & 45,2 & 34,4 \\
\hline
\end{tabular}

Note: Les calculs pour 2004 et 2010 ont été effectués en prenant en compte les résultats des élections régionales en Alsace, Aquitaine, Auvergne, Basse-Normandie, Bourgogne, Bretagne, Centre, Champagne-Ardenne, Franche-Comté, Haute Normandie, Île de France, Languedoc-Roussillon, Limousin, Lorraine, Midi-Pyrénées, Nord Pas de Calais, Pays de la Loire, Picardie, Poitou-Charente, Provence-Alpes-Côte d'Azur, Rhône-Alpes.

Les calculs pour 2015 ont été effectués en prenant en compte les résultats des élections régionales dans les nouvelles régions : Auvergne-Rhône Alpes, Bourgogne-Franche-Comté, Bretagne, Centre-Val de Loire, Grand est, Hauts-de-France, Île de France, Normandie, Nouvelle Aquitaine, Occitanie, Pays de la Loire, Provence-Alpes-Côte d’Azur.

(1) Ce chiffre a été obtenu en prenant en compte les résultats du premier tour.

(2) Ce chiffre correspond au pourcentage de votes obtenus par des coalitions / partis non construites autour du PS et de LR au second tour. 
Dans le cas de la France (Table 3), l'écart moyen entre les deux principaux partis ou coalitions a tendu à se réduire en 2015, les élections étant devenues plus disputées : 8,2\% en moyenne contre $13,7 \%$ en 2004 et $17,7 \%$ en 2010 . Comme c'est le cas en Italie, le niveau de fragmentation est resté relativement stable avec un nombre effectif moyen de partis obtenant des voix oscillant autour de 4,5 au cours des trois élections, et huit listes en moyenne obtenant au moins un pour cent des votes au premier tour. La somme des votes obtenus par les deux principaux partis au premier tour est restée relativement constante (autour de 60\%), tout comme la somme des voix obtenues par les deux principaux partis ou coalitions au second tour (entre 85 et $87,8 \%$ ). Comme dans le cas italien, la part électorale moyenne du troisième pôle (mesurée ici par les votes obtenus par les partis ou coalitions non structurés autour du PS et de LR) s'est considérablement renforcée, passant de $12,8 \%$ à $30 \%$ en 2015. Enfin, le niveau moyen de disproportionnalité, déjà élevé en 2004 et 2010 si on le compare à l'Italie (11,3\% et 11,5\% respectivement) a pratiquement doublé en 2015 pour atteindre 22,9\%. Dans certaines régions où le Parti Socialiste a choisi de ne pas se maintenir au second tour pour éviter le risque d'une victoire du Front National (Hauts de France, Provence-Alpes-Côte d'Azur), le niveau de disproportionnalité a atteint des niveaux historiques incomparables même avec ceux que l'on peut rencontrer dans des élections organisées avec des systèmes majoritaires : 34,4\% dans les Hauts de France, $31,1 \%$ en région PACA.

Ainsi, l'analyse des statistiques descriptives tend bien à suggérer également l'existence d'une relation entre le niveau de distorsion votes/sièges enregistré à une élection donnée et la taille du troisième pôle, tandis que le lien entre disproportionnalité et niveau de compétitivité des élections n'apparait pas aussi clairement que dans les simulations.

Variables et analyses de régression

De manière à tester l'effet de la taille du troisième pôle et du degré de compétitivité des élections dans chaque région sur le niveau de disproportionnalité, nous avons conduit une régression linéaire avec correction des erreurs types en clusters. Cette correction se justifie par le fait que les erreurs types sont reliées entre elles d'un groupe d'observations à l'autre. Il faut donc procéder de façon à estimer correctement les intervalles de confiance, chaque cluster correspondant à chacune des régions françaises et italiennes et le nombre d'observations étant compris de un à trois. Comme nous l'avons déjà mentionné, cette régression s’intéresse avant tout aux différences de disproportionnalité constatées d'une région à l'autre. 
La variable dépendante est l'indice des moindres carrés de Gallagher, c'est-à-dire la mesure la plus communément utilisée pour calculer la disproportionnalité entre votes et sièges. L'unité d'observation est celle de la région pour une année électorale donnée. La première variable indépendante mesure l'écart entre les deux partis / coalitions arrivés en tête, en points de pourcentage. Pour l'Italie, cet écart a été calculé en prenant en compte les résultats obtenus dans la part majoritaire, tandis que pour le cas français nous avons utilisé les résultats du second tour. Dans chaque cas, nous avons pris en compte le lieu de la compétition où se décidait l'attribution du bonus majoritaire. La deuxième variable indépendante est la taille du troisième pôle, c'est-àdire le nombre de votes obtenus par des partis ou coalitions qui ne sont pas liées au centre-droit et au centre-gauche en Italie dans la part majoritaire, et le score obtenu par les partis ou coalitions qui ne sont pas conduites par le Parti Socialiste et l'UMP puis LR au second tour en France.

Une série de variables de contrôle ont également été ajoutées, reliées soit au format de la compétition partisane soit au type de système électoral utilisé dans chaque région. La première variable de contrôle est le niveau de fragmentation du système partisan mesuré par le nombre effectif de partis politiques, calculé à partir des résultats électoraux dans la partie proportionnelle de liste en Italie et du premier tour de scrutin en France. Nous avons aussi contrôlé l'effet de la taille du parti principal, à partir des résultats des listes en Italie et du premier tour en France. La logique derrière l'inclusion de ces deux variables de contrôle consiste à considérer que l'effet de la taille du troisième pôle et de la compétitivité dans chaque scrutin régional pourrait être tempéré par le niveau de fragmentation partisane. Finalement, une dernière variable de contrôle a été ajoutée pour contrôler pour le type de système électoral utilisé dans chaque région, divisée en trois catégories : une pour le système électoral français, une pour les régions italiennes utilisant le système Tatarella «standard », et une pour les régions utilisant une variable de ce système, comme évoqué plus haut. Il était en effet indispensable de prendre en compte le fait que les systèmes électoraux régionaux ont des effets différenciés sur le niveau de disproportionnalité en France et en Italie du fait des modalités différentes d'attribution du bonus, rendant le système français «mécaniquement» plus disproportionnel (Bedock et Sauger 2014). Des tests ont été conduits pour vérifier l'absence de multicollinéarité des variables indépendantes et des variables de contrôle (Annexes). Il s'avère que le niveau de fragmentation est très fortement corrélé avec la taille du principal parti, nous conduisant à tester ces deux variables dans des modèles séparés. Il existe également une corrélation modérée $(0.28, \mathrm{p}=0.01)$ entre l'écart entre les deux principales coalitions et la taille du parti principal, mais n'étant pas de nature à poser des problèmes statistiques. 
Table 4. Résultats de la régression linéaire (variable dépendante : indice des moindres carrés)

\section{Modèle 1 Modèle 2}

Écart entre les deux principaux

partis ou coalitions

$-0.127 * * * \quad-0.145^{* * *}$

$(0.00) \quad(0.00)$

Taille du troisième pôle

$\begin{array}{cc}0.260^{* * *} & 0.262^{* * *} \\ (0.00) & (0.00)\end{array}$

Nombre effectif de partis en compétition

Taille du principal parti

Système électoral (cat. de ref. : France)

\begin{tabular}{lcc} 
Tatarella & $-4.737^{* * *}$ & $-4.721^{* * *}$ \\
& $(0.00)$ & $(0.00)$ \\
Variation & $-4.676^{* * *}$ & $-4.791^{* * *}$ \\
& $(0.00)$ & $(0.00)$ \\
& & \\
& & \\
Constant & $12.312^{* * *}$ & $8.093^{* * *}$ \\
& $(0.00)$ & $(0.00)$ \\
N & & \\
R-square & 99 & 99 \\
\hline \hline
\end{tabular}

p-values entre parenthèses

$* \mathrm{p}<0.05,{ }^{* *} \mathrm{p}<0.01, * * * \mathrm{p}<0.001$

Erreurs-types en clusters

Les résultats sont résumés dans deux modèles séparés (Table 4). Ils confirment l'un comme l'autre les deux hypothèses et montrent l'existence d'un effet indépendant du niveau de

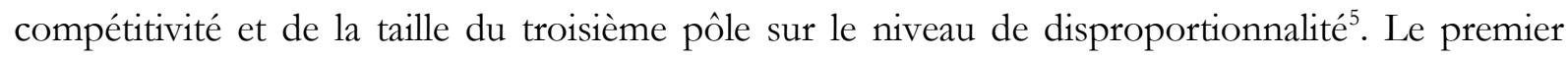
modèle montre que quand l'écart entre les deux partis ou coalitions correspond à 20 points de pourcentage, le niveau de disproportionnalité enregistré toutes les autres variables étant tenues constantes est réduit de 2,5 points, les résultats étant statistiquement très significatifs. Quand on contrôle non plus par la fragmentation, mais par la taille du principal parti, l'effet est plus

${ }^{5}$ Des régressions, non reportées ici, ont été effectuées pour vérifier l'existence d'un éventuel effet d'interaction entre le niveau de compétitivité et le troisième pôle, qui se sont révélées non concluantes. 
important: quand l'écart entre les deux coalitions est de 20 points de pourcentage, la disproportionnalité vote/sièges diminue de trois points. En d'autres termes, lorsque l'écart entre les partis ou coalitions qui cherchent à obtenir le bonus majoritaire est important, le niveau de distorsion entre votes et sièges est plus faible. Ce résultat confirme que, toutes choses égales par ailleurs, la pénalité pour les partis qui n'obtiennent pas le bonus est plus importante lorsque la « course » électorale est serrée. Les deux modèles confirment également l'effet indépendant de la taille du troisième pôle sur la disproportionnalité, avec des coefficients très comparables dans les modèles 1 et 2. Cet effet est non seulement statistiquement significatif, mais également substantiel : lorsque le poids électoral du troisième pôle passe de 15 à 35\% des votes, le niveau de disproportionnalité prévu par les deux modèles augmente de 5,2 points. Le déclin de la bipolarisation de la compétition électorale en France et en Italie s'accompagne donc clairement d'une montée substantielle de la distorsion entre votes et sièges, tous les autres facteurs étant tenus constants. Les variables de contrôle montrent quant à elles que ni le nombre effectif de partis en compétition ni la taille du principal parti n'ont d'effet propre sur le niveau de disproportionnalité enregistré. En revanche, et comme attendu, le système électoral utilisé a un effet statistiquement significatif sur le niveau de disproportionnalité enregistré : les modèles montrent que la distorsion votes/sièges est respectivement de 4,7 points inférieure en Italie que l'on utilise le système Tatarella ou une variante (modèles 1 et 2).

\section{Conclusion : quelles conséquences pour la représentation?}

Pour conclure cet article, il est utile de faire un pas de côté pour illustrer la portée des résultats empiriques mis en avant à partir d'un exemple concret. En effet, ce qui est en jeu ici ne concerne pas seulement les quelques aficionados de systèmes électoraux complexes, mais bien plus fondamentalement la question de la qualité de la représentation politique dans des systèmes politiques qui cherchent à forcer une logique majoritaire par le biais du système électoral dans des systèmes partisans profondément fragmentés, tripolaires et tendant à se morceler encore davantage. Dans les Hauts-de-France (nouvelle région française composée de l'ancienne région du Nord-Pas-de-Calais et de la Picardie), en 2015, à l'occasion du premier tour, seules trois listes sont parvenues à passer le seuil de 10\% permettant l'accès au second tour : la liste d'Union de la gauche (conduite par le Parti Socialiste) avec 17,3\% des suffrages exprimés, la liste d'union de la droite (conduite par LR et les centristes de l'UDI) avec 26,3\% des voix, et le Front National qui obtient $41 \%$ des votes. Lors du second tour, le Parti Socialiste choisit de se retirer de la compétition pour éviter l'élection de Marine Le Pen comme présidente de la région sous 
l'étiquette FN. Par conséquent, la liste LR/UDI parvient à emporter la région contre le Front National avec 56,4\% des voix contre $43,6 \%$ pour le Front National. La gauche a été totalement balayée du nouveau conseil régional, alors que la droite obtient 116 sièges sur 170 (68\% des sièges) avec un quart des votes au premier tour, le reste des sièges étant attribués au Front National. Le niveau de disproportionnalité enregistré a donc atteint 34,4\%, un niveau de disproportionnalité totalement incomparable avec le plus majoritaire de tous les systèmes électoraux majoritaires. Le cas des Hauts-de-France, tout comme celui par exemple de la Provence-Alpes-Côte d'Azur, illustre d'une manière assez drastique comment la combinaison d'un troisième pôle extrêmement puissant et considéré comme dangereux pour la démocratie par les autres partis en compétition et une compétition électorale serrée peut amener une distorsion votes/sièges qui pose véritablement question quant à la représentation politique des différentes sensibilités politiques.

Au-delà de cet exemple précis, les cas italien et français remettent en question la justification principale avancée pour défendre les systèmes proportionnels avec bonus, à savoir leur capacité à combiner gouvernabilité et représentativité. Le simple fait que le même système électoral puisse, d'une élection à l'autre et dans la même région du simple au triple voire davantage selon la configuration électorale est problématique dans la mesure où cela implique que les effets de ces systèmes sont profondément contingents au format de la compétition électorale. En d'autres termes, leurs effets épousent les évolutions de la compétition plus qu'ils ne contribuent à la structurer. Il est aussi à noter que dans le cas italien comme dans le cas français, les dernières péripéties électorales sont marquées non seulement la consolidation du Mouvement Cinq Etoiles et du Front National, mais aussi par l'émergence de nouvelles forces politiques puissantes (dont En Marche ou la France Insoumise pour ne citer que la France). De plus, les forces électorales ayant dominé la compétition (centre-gauche et centre-droit en Italie, PS et LR en France) sont clairement en perte de vitesse face à une recomposition partisane dont l'ampleur et les conséquences restent encore à évaluer. Dans un cas comme dans l'autre, les systèmes électoraux cherchant explicitement à «fabriquer» des majorités apparaissent donc comme ajoutant un élément d'incertitude important et comme entraînant dans certaines configurations électorales particulières des niveaux de disproportionnalité que l'on peut considérer comme inacceptables, si l'on considère qu'un système électoral «juste» a pour but de concilier « représentation et responsabilité » (Ganghof 2016). Il est aussi utile de rappeler que dans le cas français, lorsque le nouveau système électoral est adopté au Parlement en 2003, celui-ci est créé très explicitement dans le but de permettre la formation de majorités stables sans le Front 
National ${ }^{6}$, pariant donc sur le fait que ce parti ne serait jamais en mesure de l'emporter seul au second tour. A la lumière des développements récents, on peut se demander si la promotion de tels systèmes électoraux pour écarter explicitement certaines forces ne relève pas de manœuvres d'apprenti sorcier.

Plus encore, les résultats de cet article remettent en question la capacité de ce type de systèmes électoraux à remplir l'un des principaux rôles traditionnellement assignés aux institutions, à savoir la réduction de l'incertitude. Douglass North considère par exemple que « les institutions réduisent l'incertitude en fournissant une structure à la vie de tous les jours » (1990, 3). Elles sont donc supposées assurer une certaine stabilité et une certaine prédictibilité dans les interactions humaines. Si l'on applique ce cadre à la question spécifique des systèmes électoraux, cela implique que l'un des rôles majeurs des tout système électoral est de stabiliser un certain nombres de conséquences prévisibles sur la répartition entre votes et sièges qui est à la fois la conséquence de ses effets mécaniques, psychologiques, et des comportements récurrents adoptés par les acteurs politiques qui les utilisent. Ce qui est au contraire très frappant dans les deux cas examinés dans cet article, c'est que le même système électoral implique des conséquences très différentes d'une élection à l'autre au sein de la même région tout en ayant une capacité de plus en plus limitée à concilier représentation et gouvernabilité. Plus largement, cet article contribue à la connaissance des effets des modes de scrutin en démontrant que ceux-ci peuvent être, notamment dans les systèmes complexes, extrêmement contingents au format de la compétition électorale circonscription par circonscription. Dans la mesure où plusieurs pays européens font face à une fragmentation croissante de leurs systèmes partisans, il est urgent de mieux comprendre comment ces mutations ont une incidence sur la disproportionnalité dans divers système électoraux, et donc sur la capacité à résoudre la complexe équation liant représentation et responsabilité.

${ }^{6}$ On peut en trouver les signes lors des débats à l'Assemblée nationale et au Sénat sur la loi relative à l'élection des conseillers régionaux of 2003: http://www.assembleenationale.fr/12/dossiers/conseillers_regionaux.asp (consulté on the 25 mai 2017). 


\section{Bibliographie}

Baldini, Gianfranco. 2013. «Don't Count Your Chickens before They're Hatched: The 2013 Italian Parliamentary and Presidential Elections ». South European Society and Politics 18 (4): 473-97.

Bedock, Camille, et Nicolas Sauger. 2014. «Electoral Systems with a Majority Bonus as unconventional Mixed Systems ». Representation 50 (1): 99-112.

Benoit, Kenneth. 2002. "The endogeneity problem in electoral studies: a critical re-examination of Duverger's mechanical effect ». Electoral Studies 21 (1): 35-46.

. 2007. «Electoral Laws as Political Consequences: Explaining the Origins and Change of Electoral Institutions ». Annual Review of Political Science 10: 363-90.

Bochsler, Daniel. 2012. «A Quasi-Proportional Electoral System 'Only for Honest Men’? The Hidden Potential for Manipulating Mixed Compensatory Electoral Systems ». International Political Science Review 33 (4): 401-20.

. 2014. «Which Mixed-Member Proportional Electoral Formula Fits You Best? Assessing the Proportionality Principle of Positive Vote Transfer Systems ». Representation 50 (1): 113 -27 .

Bochsler, Daniel, et Julian Bernauer. 2014. «Strategic Incentives in Unconventional Electoral Systems: Introduction to the Special Issue ». Representation 50 (1): 1-12.

Boix, Carles. 1999. "Setting the rules of the game: the choice of electoral systems in advanced democracies ». American Political Science Review 93 (3): 609-624.

Bornschier, Simon, et Romain Lachat. 2009. «The Evolution of the French Political Space and Party System ». West European Politics 32 (2): 360-83.

Carey, John M, et Simon Hix. 2011. "The Electoral Sweet Spot: Low-Magnitude Proportional Electoral Systems ». American Journal of Political Science 55 (2): 383-97.

Chiaramonte, Alessandro. 2010. «Dal bipolarismo frammentato al bipolarismo limitato? evoluzione del sistema partitico italiano. » In Proporzionale se vi pare. Le elezioni politiche del 2008, édité par Roberto D’Alimonte et Alessandro Chiaramonte, 203-28. Bologna: Il Mulino.

Chiaramonte, Alessandro, et Vincenzo Emanuele. 2013. «Volatile and tripolar: the new Italian party system ». In The Italian General Elections of 2013: A dangerous stalemate?, édité par Lorenzo De Sio, Vincenzo Emanuele, Nicola Maggini, et A. Paparo, 63-68. Rome: Cise.

Colomer, Josep. 2005. «It's Parties that Choose Electoral Systems (or, Duverger's Laws Upside Down)». Political studies 53 (1): 1-21.

Conti, Nicolò, et Vincenzo Memoli. 2015. «The Emergence of a New Party in the Italian Party System: Rise and Fortunes of the Five Star Movement ». West European Politics 38 (3): 51634.

Cox, Gary. 1997. Making Votes Count. Cambridge: Cambridge University Press.

D'Alimonte, Roberto. 2005. "Italy: a case of fragmented bipolarism ». In The Politics of Electoral Systems, par Michael Gallagher et Paul Mitchell, 253-76. Oxford: Oxford University Press. 
D'Alimonte, Roberto. 2015. "The new Italian electoral system: majority-assuring but minorityfriendly ». Contemporary Italian Politics 7 (3): 286-92.

D’Alimonte, Roberto, et Stefano Bartolini, éd. 2002. Maggioritario finalmente? La transižione elettorale 1994-2001. Bologna: Il Mulino.

Di Virgilio, Aldo, Bernard Dolez, et Annie Laurent. 2016. "Systèmes électoraux " complexes », coordination pré-électorale complexe. Une comparaison France-Italie ». Revue internationale de politique comparée 22 (3): 343-70.

Duverger, Maurice. 1951. Les partis politiques. Paris: Armand Colin.

Ehrhard, Thomas. 2016. «Le Front national face aux modes de scrutin : entre victoire sous conditions et influences sur le système partisan ». Pouvoirs, n 157 (avril): 85-103.

Farrell, David M., et Richard S. Katz. 2014. "Assessing the Proportionality of the Single Transferable Vote ». Representation 50 (1): 13-26.

Ferrara, Federico, Erik S. Herron, et Misa Nishikawa. 2005. Mixed Electoral Systems: Contamination and Its Consequences. First Edition. Basingstoke, New York: Palgrave Macmillan.

Gallagher, Michael. 1991. «Proportionality, disproportionality and electoral systems ». Electoral Studies 10 (1): 33-51.

Ganghof, Steffen. 2016. "Reconciling Representation and Accountability: Three Visions of Democracy Compared ». Government and Opposition 51 (2): 209-33.

Gougou, Florent, et Simon Labouret. 2013. « La fin de la tripartition ?: Les recompositions de la droite et la transformation du système partisan ». Revue française de science politique 63 (2): 279. doi:10.3917/rfsp.632.0279.

Gougou, Florent, et Simon Persico. 2017. «A new party system in the making? The 2017 French presidential election ». French Politics 15: 303-21.

Grunberg, Gérard, et Étienne Schweisguth. 2003. «Chapitre 13. La tripartition de l'espace politique ». Chroniques électorales, 339-62.

Haegel, Florence. 2007. Partis politiques et système partisan en France. Paris : Presses de Sciences po.

Herron, Erik S, et Misa Nishikawa. 2001. "Contamination effects and the number of parties in mixed-superposition electoral systems ». Electoral Studies 20 (1): 63-86.

Lijphart, Arend. 1994. Electoral systems and party systems: a study of twenty-seven democracies, 1945-1990. Oxford: Oxford University Press.

Manow, Philip. 2011. "The Cube Rule in a Mixed Electoral System: Disproportionality in German Bundestag Elections ». West European Politics 34 (4): 773-94.

Massicotte, Louis, et André Blais. 1999. «Mixed electoral systems: a conceptual and empirical survey ». Electoral Studies 18 (3): 341-66.

Moser, Robert G., et Ethan Scheiner. 2004. «Mixed electoral systems and electoral system effects: controlled comparison and cross-national analysis ». Electoral Studies 23 (4): 57599.

North, Douglas. 1990. Instititutions, Institutional Change and Economic Performance. New York: Cambridge University Press.

Pasquino, Gianfranco, et Marco Valbruzzi. 2013. «Post-electoral politics in Italy: institutional problems and political perspectives ». Journal of Modern Italian Studies 18 (4): 466-84. 
Passarelli, Gianluca. 2014. "Electoral Law(s) and Elections in the Italian Second Republic: The 2013 Landmark (?)’ ». Polis (Italy) 28 (1): 107-22.

Rae, Douglas W. 1969. The political consequences of electoral laws. New Haven: Yale University Press.

Riera, Pedro. 2013. «Electoral Coordination in Mixed-Member Systems: Does the Level of Democratic Experience Matter? » European Journal of Political Research 52 (1): 119-41.

Rokkan, Stein. 1970. Citizens, Elections, Parties. Approaches to the comparative study of the processes of development. New York: David McKay.

Sauger, Nicolas. 2007. «Un système électoral vecteur d'instabilité ? L'impact du système électoral sur la structuration du système partisan sous la Cinquième République ». In Les partis politiques en France, édité par Florence Haegel, 359-391. Paris: Presses de Sciences Po.

Shugart, Matthew S., et Martin P. Wattenberg, éd. 2001. Mixed-member electoral systems: the best of both worlds. Oxford: Oxford University Press.

Taagepera, Rein. 2007. Predicting Party Sizes: The Logic of Simple Electoral Systems. Oxford : Oxford University Press.

Taagepera, Rein, et Matthew S. Shugart. 1989. Seats and votes: the effects and determinants of electoral systems. New Haven: Yale University Press.

Tronconi, Filippo. 2015. «Bye-Bye Bipolarism: The 2015 Regional Elections and the New Shape of Regional Party Systems in Italy ». South European Society and Politics 20 (4): 553-71.

Tronconi, Filippo, et Christophe Roux. 2009. «The political systems of Italian regions between state-wide logics and increasing differentiation ». Modern Italy 14 (2): 151-66.

Vampa, Davide. 2015. «The 2015 Regional Election in Italy: Fragmentation and Crisis of Subnational Representative Democracy ». Regional \& Federal Studies 25 (4): 365-78.

2016. "Declining Partisan Representation at the Sub-National Level: Assessing and Explaining the Strengthening of Local Lists in Italian Municipalities (1995-2014)». Local Government Studies, février, 1-19. 